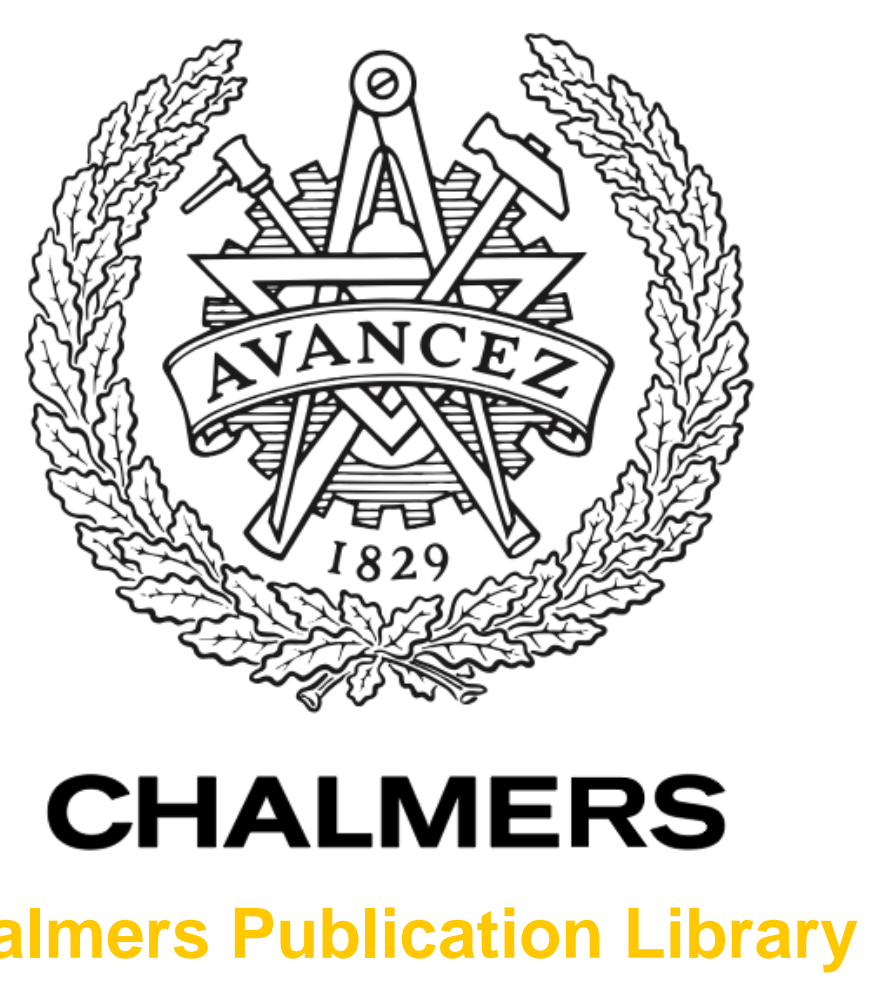

Chalmers Publication Library

\title{
Feedback Analysis in Percutaneous Bone-Conduction Device and Bone-Conduction Implant on a Dry Cranium
}

This document has been downloaded from Chalmers Publication Library $(\mathrm{CPL})$. It is the author's version of a work that was accepted for publication in:

Otology and Neurotology (ISSN: 1531-7129)

Citation for the published paper:

Taghavi, H. ; Håkansson, B. ; Reinfeldt, S. (2012) "Feedback Analysis in Percutaneous Bone-Conduction Device and Bone-Conduction Implant on a Dry Cranium". Otology and Neurotology, vol. 33(3), pp. 413â420.

http://dx.doi.org/10.1097/MAO.0b013e3182487fc8

Downloaded from: http://publications.lib.chalmers.se/publication/155866

Notice: Changes introduced as a result of publishing processes such as copy-editing and formatting may not be reflected in this document. For a definitive version of this work, please refer to the published source. Please note that access to the published version might require a subscription. 


\title{
Complete manuscript title:
}

Feedback Analysis in Percutaneous Bone Conduction Device (PBCD) and Bone Conduction Implant $(\mathrm{BCl})$ on a Dry Skull

\section{Short running head:}

Feedback in PBCD and BCI

\section{Authors' full names}

Hamidreza Taghavi ${ }^{1}$, Bo Håkansson ${ }^{1}$, Sabine Reinfeldt ${ }^{1}$, Måns Eeg-Olofsson ${ }^{2}$, Shirin Akhshijan ${ }^{1}$

\section{Highest academic degrees}

Hamidreza Taghavi (M.Sc.)

Bo Håkansson (Ph.D.)

Sabine Reinfeldt (Ph.D.)

Måns Eeg-Olofsson (M.D.)

Shirin Akhshijan (M.Sc.)

\section{Affiliations}

1. Division of Signal Processing and Biomedical Engineering, Department of Signals and Systems, Chalmers University of Technology, Göteborg, Sweden

2. ENT Department, Sahlgrenska University Hospital, Department of Otorhinolaryngology, Head and Neck Surgery, The Sahlgrenska Academy, Göteborg University, Göteborg, Sweden

\author{
Name and address for correspondence, including fax number, \\ telephone number, and e-mail address \\ Hamidreza Taghavi \\ Chalmers University of Technology, Department of Signals and Systems, 412 96, Göteborg, Sweden \\ Phone: $\quad+46317728062$ \\ Fax: $\quad+46317721782$ \\ email: taghavi@chalmers.se \\ Web: $\quad$ www.chalmers.se/s2
}

Sources of support that require acknowledgment

VINNOVA: Swedish Governmental Agency for Innovation Systems 


\begin{abstract}
Hypothesis:

The Bone Conduction Implant $(\mathrm{BCl})$ can use a higher gain setting without having feedback problems as compared with a Percutaneous Bone Conduction Device (PBCD).

\section{Background:}

The conventional PBCD is today a common treatment for patients with conductive hearing loss and single sided deafness. However there are minor drawbacks reported related to the percutaneous implant and specifically poor high frequency gain. The $\mathrm{BCl}$ system is designed as an alternative to the percutaneous system, because it leaves the skin intact and is less prone to fall into feedback oscillations thus allowing more high frequency gain.
\end{abstract}

\title{
Methods:
}

Loop gains of the Baha Classic 300 and the $\mathrm{BCl}$ were measured in the frequency range of 100 to $10000 \mathrm{~Hz}$ attached to a Skull simulator and a dry skull. The Baha and the $\mathrm{BCl}$ positions were investigated. The devices were adjusted to Full-on Gain.

\section{Results:}

It was found that the gain headroom using the $\mathrm{BCl}$ was generally $0-10 \mathrm{~dB}$ better at higher frequencies than using the Baha for a given mechanical output. More specifically, if the mechanical output of the devices were normalized at the cochlear level the improvement in gain headroom with the $\mathrm{BCl}$ versus the Baha were in the range of $10-30 \mathrm{~dB}$.

\section{Conclusion:}

Using a $\mathrm{BCl}$, significantly higher gain setting can be used without feedback problems as compared with using a PBCD.

Key words: Percutaneous Bone Conduction Device, Baha, Bone Conduction Implant, feedback, implantable transducer, sound radiation, loop gain, stability, gain headroom. 


\section{Introduction}

Patients who are suffering from conductive and mixed hearing loss can sometimes not be rehabilitated by conventional air conduction (AC) hearing aids. If the sensorineural hearing is sufficient, a bone conduction (BC) hearing aid can be used for transmitting sound to the cochlea. In the 1980s a new generation of BC hearing aid called the Bone Anchored Hearing Aid was introduced which is a Percutaneous Bone Conduction Device (PBCD). PBCD is an important treatment for patients who cannot be rehabilitated by AC hearing aids (1-3) due to chronic otitis and malformations of the ear canal and the middle ear. PBCDs are also fitted in patients having single sided deafness (SSD) to reduce the head shadow effect (4-6). Figure 1 illustrates the principal design of the PBCD. Although very good rehabilitation results with the PBCD are reported (3), there are some known complications related to both the soft tissue around the implant and the implant anchorage to the bone (7-11). Even though some complications seem to decrease with new implant systems (12), one main issue is that it needs life-long daily care. Also, the bone-anchored fixture can be lost spontaneously or as a result of trauma, especially in children (13-14). Lastly, percutaneous bone conduction devices are known to have limited gain due to feedback problems. Feedback oscillations occur when increasing the gain over a certain point as sound is radiated back from skull bone and housing to the microphone. This is in fact an inherent limiting factor for PBCD gain at higher frequencies.

A novel Bone Conduction Implant ( $\mathrm{BCl}$ ) has been proposed and developed as an alternative to the percutaneous bone conduction hearing device (15-17). It is shown in Figure 2 that the $\mathrm{BCl}$ contains two main parts; a) the audio processor which includes a plastic housing with battery compartment, digital and analog signal processing units, tuned power amplifier and transmitter radio frequency (RF) coil; and b) the implanted unit called $\mathrm{BCl}$ Bone Bridge that consists of RF receiver coil, filters and the $\mathrm{BCl}$ transducer. In Figure 3 it is illustrated that the speech signal is amplitude modulated and transmitted wirelessly via an inductive RF link through the intact skin to the implant. The audio processor housing and the implanted coil are aligned by a retention system with a permanent magnet in each unit. As this force is only for retention and not for coupling/transmitting bone conducted sound, it is smaller than the retention force in conventional bone conduction devices. It is expected that such retention force can be less than 10 times the mass of the external device (approximately $10 \mathrm{~g}$ ) which means approximately $1 \mathrm{~N}$. Furthermore, this force is distributed on a larger area, i.e. force per area will be less than in conventional bone conduction devices. Finally, this method of retention is also used in cochlear implants and middle ear implants like Vibrant Sound Bridge. The $\mathrm{BCl}$ transducer has approximately half the size relative of a bone anchored hearing device transducer and is permanently implanted under the skin. It is attached to the skull via a flat surface 
of the housing held in place with a clamping bar construction using miniature orthopedic (Champy) screws. This transducer has a high frequency resonance which boosts the speech signal in the high frequency range. This boost might be beneficial in hearing speech cues. Also, the capsuled $\mathrm{BCI}$ transducer uses the Balanced Electromagnetic Separation (BEST) principle. For more details about present prototypes see $(16,17)$.

The surgical procedure that has been tested in cadavers and animal models is assumed to be minimally invasive and similar to PBCD surgery. Details will be published in a future article.

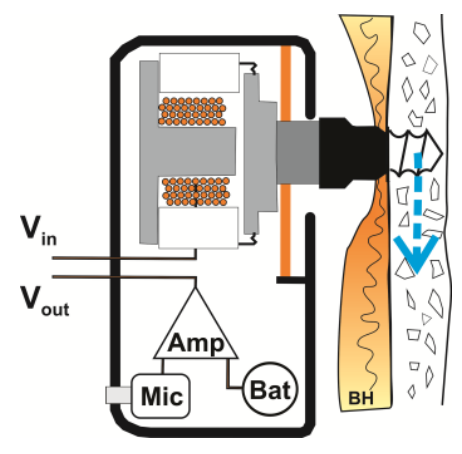

Figure 1. Principal design of a generic percutaneous Bone Conduction Device (PBCD) with a screw attachment to the skull bone. It also comprises a microphone (Mic), battery (Bat) and pre and power amplifier (Amp) that drives the bone conduction transducer. The connection between the Amp and the transducer is opened for loop gain measurements.

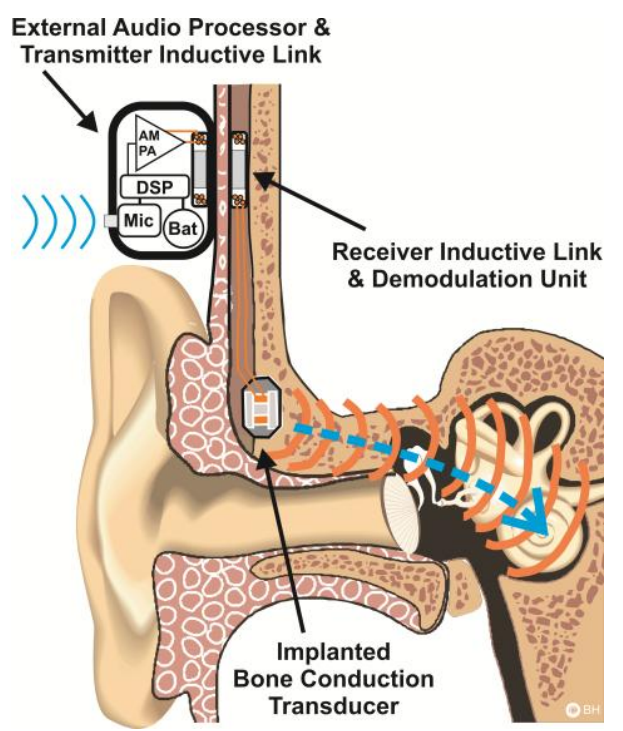

Figure 2. BCl system with an implanted and capsuled bone conduction transducer with a flat surface contact to the skull bone. It comprises also a receiver coil and an externally worn audio processor. The audio processor comprises a digital signal processor (DSP) and an amplitude modulation (AM) power amplifier (PA). 


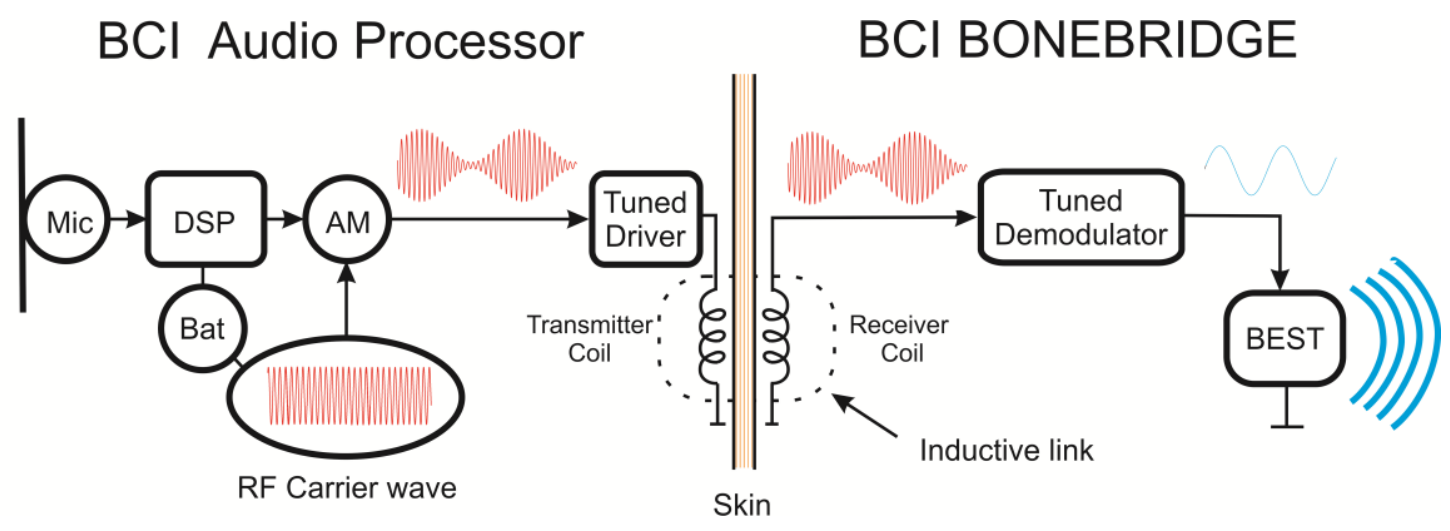

Figure 3. Principal design of the full-scale $B C l$ device. The speech signal is picked up by the microphone (Mic) and fed to the digital signal processor (DSP). It is then transmitted through the intact skin using amplitude modulation (AM) of the radio frequency (RF) carrier wave. The speech signal is extracted by the tuned demodulating unit and then is fed to the $\mathrm{BCl}$ transducer which uses the Balanced Electromagnetic Separation (BEST) principle.

\section{Aim of Study}

During development of the $\mathrm{BCl}$ and particularly in previous cadavers studies $(15,16)$, it has been noted that the $\mathrm{BCl}$ device was less prone to fall into feedback problems than the PBCD especially at higher frequencies. This finding indicates that the $\mathrm{BCl}$ can allow a higher gain setting than the $\mathrm{PBCD}$ without problems with feedback. An increased amplification especially at high frequencies can be very beneficial for speech understanding. The aim of this study is to investigate the gain headroom (how much extra gain can be provided before the device will oscillate) in the $\mathrm{BCl}$ and in a generic bone anchored hearing device.

\section{Theory and Classification of Feedback in Bone Conduction Devices}

In bone conduction devices, there are different undesirable feedback paths from the transducer to the microphone that can cause severe problems with feedback oscillations. Feedback is an inherent gain limiting factor in hearing aids due to proximity of the transducer and microphone. This undesired feedback is frequency dependent and results in ringing sounds or in worst case oscillations when a certain maximum allowable gain is exceeded. In digital signal processors for hearing aids, different algorithms are used for reducing undesired feedback in so called adaptive feedback suppression systems. These algorithms and methods are not the focus of this article.

\section{Analytical model for the PBCD}

There are basically four main types of undesired feedback paths that can occur in the PBCD. All can be defined by a feedback function dependent on frequency (f). In this paper the symbol " $\mathrm{f}$ " is regarded as the complex frequency. 
1- Structure-borne mechanical feedback $\alpha_{1}(f)$; transducer vibrations that are mechanically transmitted through the housing back to the microphone,

2- Acoustical feedback $\alpha_{2}(f)$; caused by the sound radiated from the skull bone and from the hearing aid housing which are acoustically transmitted back to the microphone,

3- Electrical feedback; voltage fluctuations in the output amplifier are electrically transmitted to the preamplifier or the microphone within the electric circuit, and finally,

4- Magnetic feedback; magnetic leakage from the transducer are magnetically transmitted to the microphone or possible a telecoil (if such is used in the vicinity of the transducer).

The combination of electrical and magnetic feedbacks is denoted as $\alpha_{3}(f)$ are considered as a single function due to the two following circumstances; a) these two feedback contributions are very low compared with the acoustical and mechanical feedback combinations; and $b$ ) it is difficult to measure their separate contributions.

Figure 4 demonstrates the block diagram of the proposed analytical model. It should be noted that the output signal "Out" from the transducer is force and the input signal "In" to the microphone is sound pressure. The virtual summation point $(\Sigma)$ corresponds to the microphone diaphragm (18).

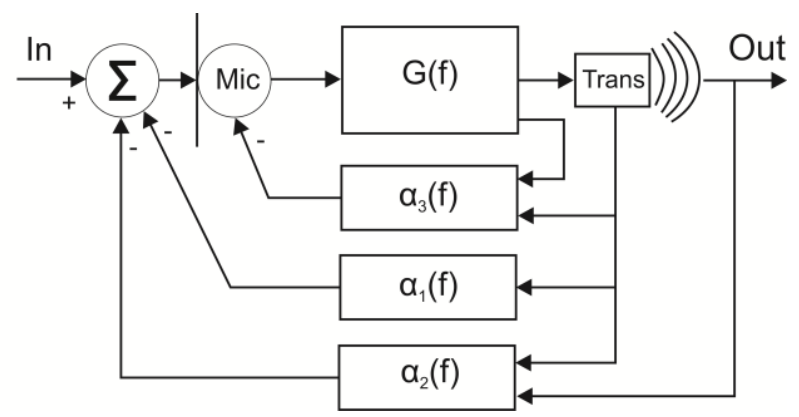

Figure 4. Proposed analytical model of the PBCD employing structure-borne mechanical $\alpha_{1}(f)$, acoustical $\alpha_{2}(f)$, and combination of electrical and magnetic $\alpha_{3}(f)$ feedbacks. $G(f)$ is the forward transfer function which is the $P B C D$ frequency response within normal operation i.e. when $\alpha_{1}=\alpha_{2}=\alpha_{3}=0$ (neglected). Microphone and transducer are denoted as Mic and Trans, respectively.

\section{Analytical model for the BCI}

Similar to the PBCD, there are four main types of feedback problems that potentially can occur in the $\mathrm{BCl}$. The difference is that the transducer is implanted in the skull bone and the intact skin is located between the audio processor housing and the implanted unit. This results in different amount of contributions for feedback pathways which are denoted as $\beta_{1}(f), \beta_{2}(f)$, and $\beta_{3}(f)$, see Figure 5 . 


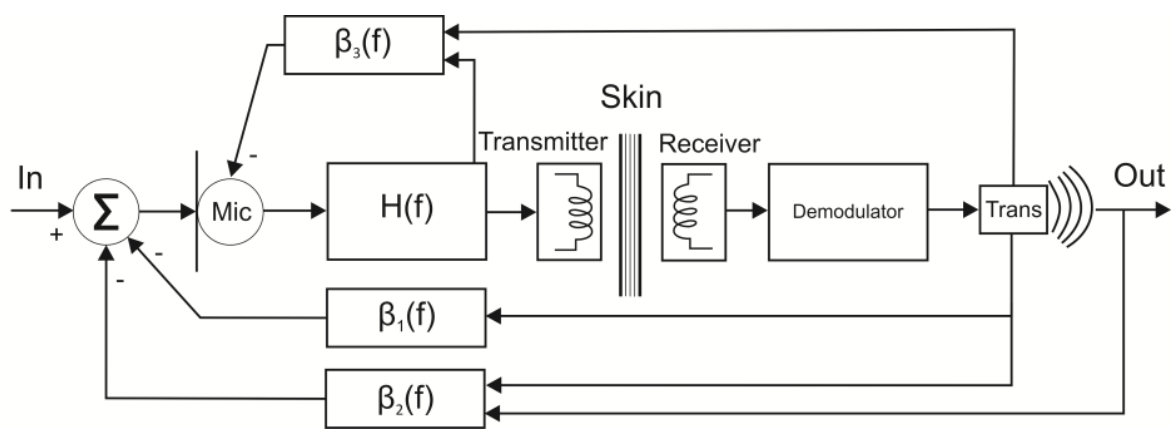

Figure 5. Proposed analytical model of the $\mathrm{BCl}$ employing structure-borne mechanical $B_{1}(f)$, acoustical $B_{2}(f)$, and combination of electrical and magnetic $B_{3}(f)$ feedbacks. $H(f)$ is the forward transfer function. Intact skin exists between the audio processor housing and the implanted unit. Microphone and transducer are denoted as Mic and Trans, respectively.

The frequency response function of a system $A(f)$ can be determined as the output signal divided by the input signal. For the $B C l$ using $H(f), \beta_{1}(f), \beta_{2}(f)$, and $\beta_{3}(f)$ we find:

$$
A(f)=\frac{H(f)}{1+\beta(\mathrm{f}) \mathrm{H}(\mathrm{f})}=\frac{H(f)}{1-T(f)}
$$

where $\beta(f)$ denotes the total $B C l$ feedback contributions and $T(f)$ is the loop gain function. The PBCD frequency response function $A(f)$ can be obtained by substituting $H(f)$ by $G(f)$ and $\beta(f)$ with $\alpha(f)$, respectively. $\alpha(f)$ and $\beta(f)$ represents the total feedback contributions. Please note that in the ideal case there is no feedback i.e. $\alpha(f)=\beta(f)=0$ which means that $T(f)=0$ and hence $A(f)=H(f)$ (or $G(f)$ ). On the other hand if $T(f)=1$ (or $0 \mathrm{~dB}$ ) a real problem occurs as $A(f)=\infty$ (infinity). This corresponds to a completely unstable system and the device will oscillate or howl at certain frequency. The only way for the patient to handle such a situation is to reduce volume control which results in reducing $T(f)$ so that $T(f)<1$.

\section{Stability condition}

In order to have a stable system, obviously $T(f)=1$ must be avoided. One practical way of saying this is that the loop gain always must be below unity i.e. $T<1$. If the loop gain is $T \geq 1$, the signal around the loop can maintain (or increase) itself on its own and thus oscillate at the frequency that $T(f)=1$. Of course the patient will experience problems such as ringing or annoying sound also when loop gain is close to $\mathrm{T}=1$. Therefore some margins to instability need to be introduced. The gain margin is the maximum additional gain that can be used before the system will start to oscillate (assuming the phase is zero degree).

The gain margin can also be interpreted as the amount by which the amplifier gain can be increased with stability maintained and before the system starts to oscillate. A rule of thumb in percutaneous 
bone conduction devices is to adjust the amplifier gain to keep 3-4 dB "gain headroom". This is done as a factory preset of gain. The term "gain headroom" will be used to denote the margin to the $0 \mathrm{~dB}$ line $(T(f)=1)$ independent of phase.

\section{Materials and Methods}

\section{Loop gain measurements (magnitude and phase)}

In this paper, to study the stability margins, the percutaneous Baha Classic 300 from Cochlear (Cochlear bone-anchored solutions; Cochlear Corporation, Sidney, Australia) and the $\mathrm{BCl}$ version 2.0 were used (19). As a generic percutaneous bone conduction device, Classic 300 was used. The reason to choose this device is that it is a linear device and does not use feedback cancellation. Since the $\mathrm{BCl}$ includes an advanced digital signal processor (DSP), all feedback cancellation algorithms were disabled and it was set for linear operation. Both devices were set to Full-on Gain. Two different positions were used on the dry skull. Position $A$ is the bone anchored hearing device standard position in the parietal bone. Position B is where the capsuled Balanced Electromagnetic Separation Transducer (BEST) was attached to the temporal bone by a static force by means of a clamping bar and two orthopedic screws (16). Loop gain measurements were made using a dry skull and a Skull simulator as loads. The Skull simulator generates a voltage proportional to the force applied at the connection point (20). The dry skull is our most important model for bone conduction testing which has a completely intact cranium with a thin layer $(1-2 \mathrm{~mm})$ polyurethane rubber attached on its inside surface and glued sutures to provide more realistic mechanical properties (21).

To be able to measure the loop gains of the Baha Classic 300 and the $\mathrm{BCl}$ the feedback loops had to be opened. In the Classic 300 the connection between the preamplifier and the power amplifier was disconnected, see Figure 1. In the $\mathrm{BCl}$, the speech signal amplifier that drives the RF power amplifier for amplitude modulation was disconnected from the RF power amplifier. When opening/breaking the feedback loop, preferably between the pre amplifier and power amplifier it was ensured that the conditions that existed prior to breaking the loop was not changed. The Classic 300 power amplifier was driven with $1 \mathrm{mV}_{\text {rms }}$ sinusoidal voltage in all loop gain measurements. This level corresponds to approximately $58 \mathrm{~dB}$ equivalent input sound pressure level (SPL). For the $\mathrm{BCl}, 7 \mathrm{mV}_{\mathrm{rms}}$ voltage was used that corresponds to approximately $56 \mathrm{~dB} \mathrm{SPL}$. It was assured that both devices were in their linear range during all measurement. A dynamic signal analyzer, Agilent 35670A, was employed to generate and analyze the signals. Automatic logarithmic swept sine in the frequency range from 100 to $10000 \mathrm{~Hz}$ was used in all measurements. All measurements were performed in a sound-insulated room. 
To measure the loop gain of the Classic 300 and the $\mathrm{BCl}$ with the dry skull as load, the dry skull was covered with $4 \mathrm{~mm}$ sticky adhesive to simulate the skin and subcutaneous soft tissues on the measuring side. It was also used to maintain a clearance between the transmitter and receiver coils in the $\mathrm{BCl}$. Figure 6 illustrates the measurement set-ups for the Classic 300 and the $\mathrm{BCl}$ devices. Summary of measurements performed:

1. Baha on dry skull, Pos A

2. Baha on Skull simulator

3. $\mathrm{BCl}$ on dry skull, Pos B

4. $\mathrm{BCl}$ on Skull simulator

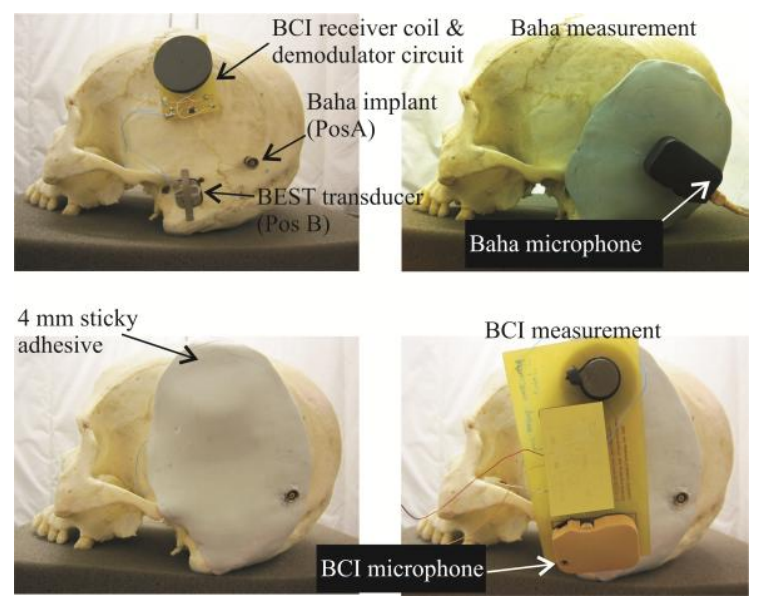

Figure 6. Measurement set-ups of the left side of the skull for the Baha Classic 300 and the BCl devices with $4 \mathrm{~mm}$ sticky adhesive used as skin between transmitter and receiver coils of the $B C I$. In the $B C I$ set-up, the microphone was located on top of the transducer for worst-case acoustical feedback effect.

In addition, to investigate the contribution of acoustical feedback only, loop gain measurements were done in all conditions with microphone inlet obstructed by sticky adhesive. Also, noise floor measurements were done in all conditions in order to investigate the certainty of the measured signal levels.

\section{Results}

\section{Loop gain measurements of the PBCD}

In Figure 7, the loop gain magnitudes and unwrapped phase diagrams of the PBCD on two different loads are presented. 
Obviously the gain headroom to the critical $0 \mathrm{~dB}$ line is small and in the range of 3-4 $\mathrm{dB}$ for the PBCD (see black solid line). By occluding the microphone inlet of the PBCD the gain headroom to the $0 \mathrm{~dB}$ line is significantly increased for frequencies from 500 to $7000 \mathrm{~Hz}$, see dashed line. This means that the dominating feedback path in the PBCD attached to the dry skull is the acoustical feedback for these frequencies.

As a Skull simulator will not reflect the radiation of sound similar to the skull bone such measurement was also performed. Clearly the Skull simulator loop gain has higher gain headroom above $500 \mathrm{~Hz}$. This means that the acoustical feedback has less contribution on a Skull simulator than on a dry skull. Obviously, in Figure 7 the noise floor curve shows that the measurements were all performed with a confident signal to noise ratio.
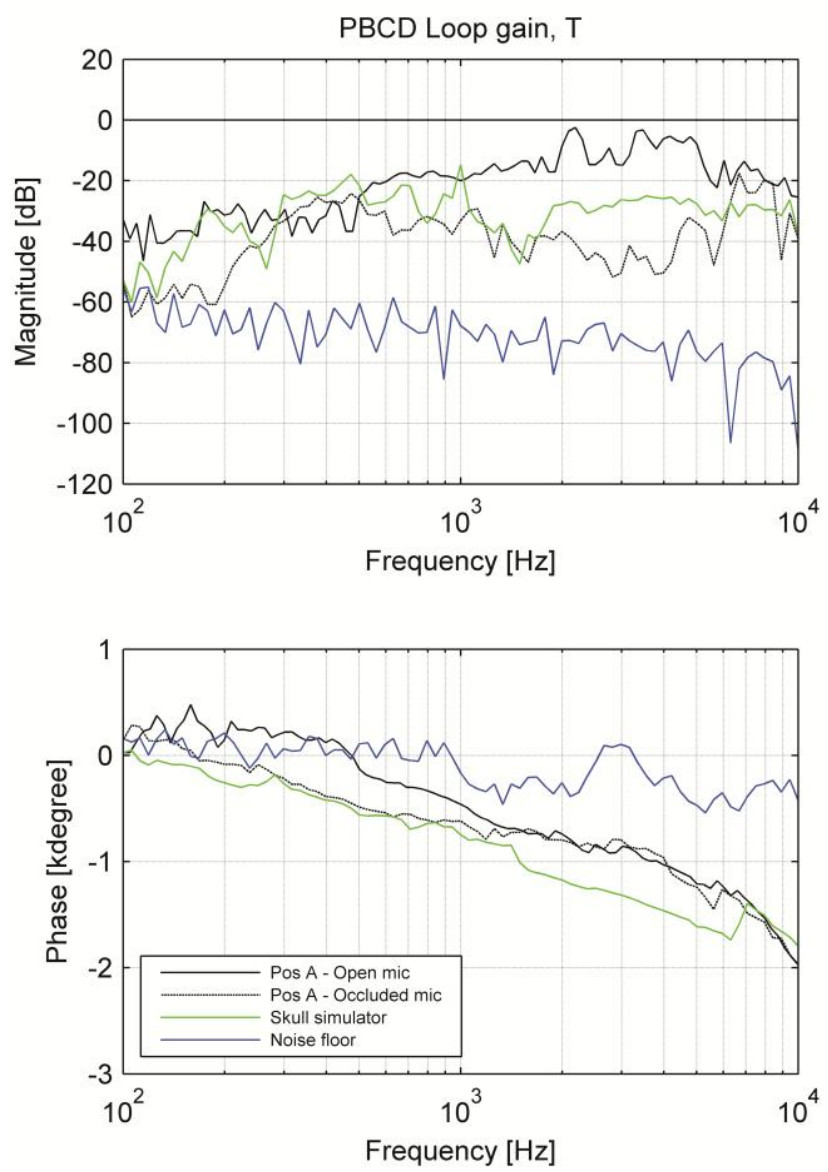

Figure 7. PBCD loop gain magnitude and phase on POS A on dry skull and Skull simulator and noise floor (solid lines). Also, the result when the microphone was occluded is shown (dashed line). Note that the critical magnitude of $T=1$ here corresponds to $T=0 \mathrm{~dB}$. 


\section{Loop gain measurements of the BCI}

Figure 8 presents the magnitude and unwrapped phase diagrams of the $\mathrm{BCl}$ device on two different loads; dry skull Pos B and Skull simulator. Obviously, the gain headroom in the $\mathrm{BCl}$ is considerably higher than in the PBCD (approximately $12 \mathrm{~dB}$ or more). Skull simulator measurement shows higher gain headroom than Pos B in frequency range of 2000 to $5000 \mathrm{~Hz}$. This is due to the "no-radiation" characteristic of the Skull simulator.
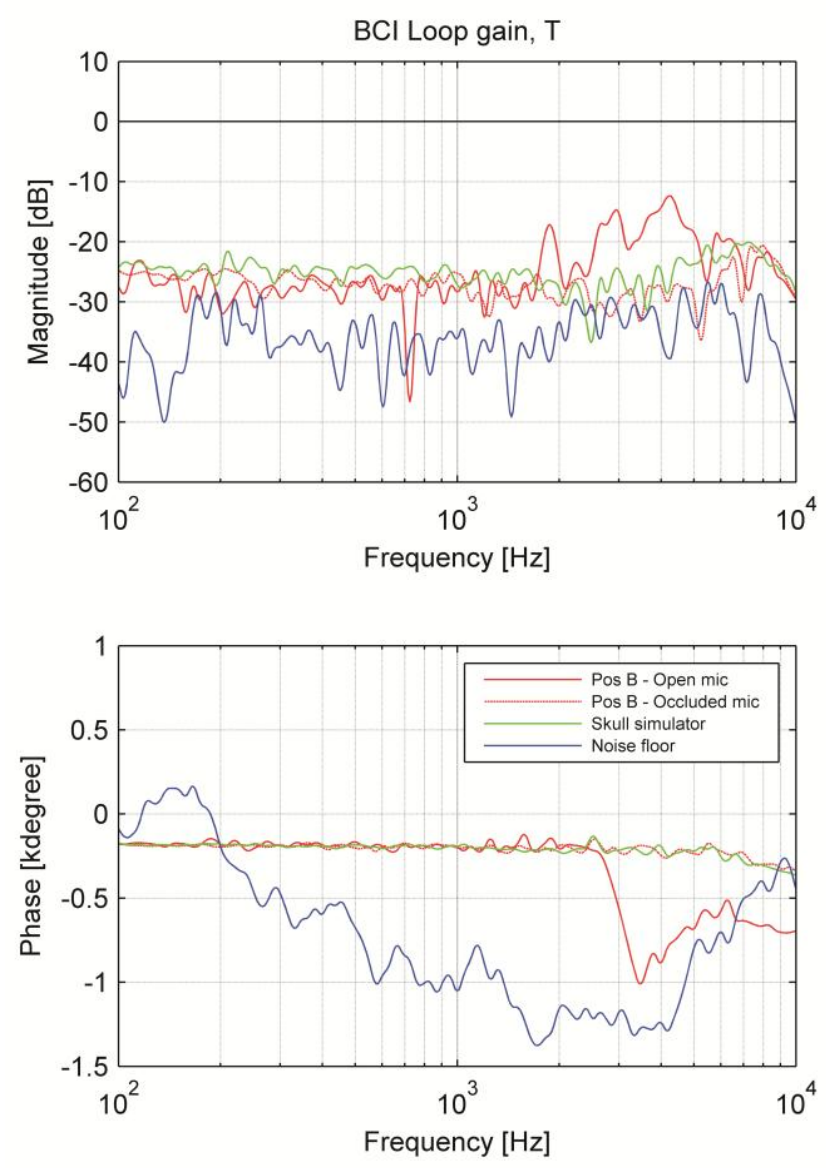

Figure 8. BCl loop gain magnitude and phase on Pos B on dry skull and Skull simulator and noise floor (solid lines). Also, the result when the microphone was occluded is shown (dashed line). Note that the critical magnitude of $T=1$ here corresponds to $T=0 \mathrm{~dB}$.

The loop gain magnitude and unwrapped phase diagrams of the $\mathrm{BCl}$ in Pos $\mathrm{B}$ when the microphone inlet was occluded was similar to the result when the $\mathrm{BCl}$ was attached to the Skull simulator, see Figure 8. Comparison to the open microphone condition, it can be concluded that the feedback is dominated by electrical feedback for all frequencies and all conditions tested except for approximately 2000 to $5000 \mathrm{~Hz}$ where the acoustical feedback is dominating. This statement is based on that the phase is not sloping for all frequencies except for 2000 to $5000 \mathrm{~Hz}$. 
Noise floor curve shows that the signal levels measured during the tests were at a sufficient high level. The noise floor is higher for the $\mathrm{BCl}$ than for the $\mathrm{PBCD}$ and that is in consequence of the difference between the opening points of the loops in the devices. In the $\mathrm{BCl}$ it is made in a later stage which then results in more microphone noise at the measured output than in the PBCD case, simply because that the noise is amplified more. This difference does not affect the loop gain measurement per se.

Figure 9 shows a comparison of the $\mathrm{PBCD}$ and the $\mathrm{BCl}$ loop gain magnitudes as they were measured and with their frequency responses as presented in Figure 10. The PBCD has a minimum of $3 \mathrm{~dB}$ gain headroom in the loop gain magnitude to avoid feedback problems. It has 0-5 dB higher gain headroom than the $\mathrm{BCl}$ below $500 \mathrm{~Hz}$. In contrast, in the important frequency range from 500 to $10000 \mathrm{~Hz}$, the $\mathrm{BCl}$ device obtains 0-25 dB more gain headroom than the PBCD, but there are big frequency variations as expected because of the acoustical feedback is present. The gain headroom in the critical frequency (close to the $0 \mathrm{~dB}$ line) is $3 \mathrm{~dB}$ for the $\mathrm{PBCD}$ and $12 \mathrm{~dB}$ for the $\mathrm{BCI}$.

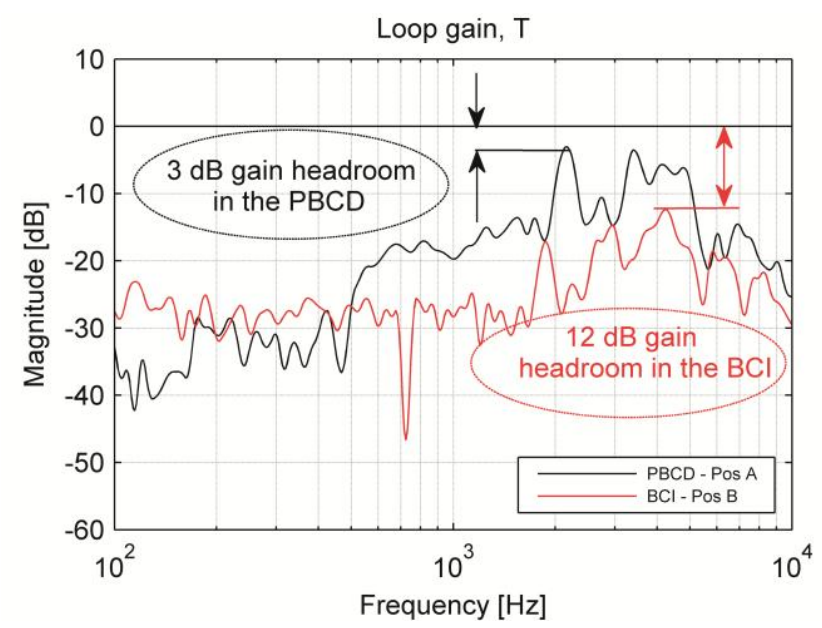

Figure 9.The $P B C D$ and the $B C l$ loop gain magnitudes on dry skull. The Baha Classic 300 was positioned in Pos $A$ and the BEST transducer was implanted in Pos $B$ for the $B C l$ device.

\section{Discussion}

\section{Compensating for identical mechanical output}

It has been discussed that there might be a $10-15 \mathrm{~dB}$ output loss of the $\mathrm{BCl}$ due to the inductive $\mathrm{RF}$ link across the skin $(15,16)$. If the forward gain magnitude of the device decreases, the loop gain magnitude will also decrease with the same amount. Therefore, the $\mathrm{BCl}$ device loop gain magnitude should be compensated with the gain difference between the PBCD output force level (OFL) and the $\mathrm{BCI}$ OFL to yield the same mechanical output. Linear spectrum magnitudes of the Baha Classic 300 
and the $\mathrm{BCl}$ were therefore measured on Skull simulator in the anechoic chamber while the input sound pressure kept constant at $60 \mathrm{~dB}$ SPL which is in the linear range of both devices, see Figure 10 . A $4 \mathrm{~mm}$ clearance (silicone sheet) was used between transmitter and receiver coils in the $\mathrm{BCl}$. As expected in most frequency ranges, the PBCD has higher output force than the $\mathrm{BCl}$ of $10-15 \mathrm{~dB}$ (attributed to inductive transmission loss) except in the frequency range from 4000 to $6500 \mathrm{~Hz}$ where the $\mathrm{BCl}$ has even a higher output force. It should be pointed out that the $\mathrm{BCl}$ transducer has a high frequency boost resonance that has been described by Håkansson et al. (16) and that not exist in the Classic 300 transducer. In Figure 11, the PBCD loop gain is presented together with the compensated $\mathrm{BCl}$ loop gain for identical mechanical output. The compensated $\mathrm{BCl}$ loop gain is now more similar to the loop gain of the PBCD in the mid frequency range, but there is still an advantage of $10 \mathrm{~dB}$ higher gain headroom compared to the PBCD in the 3000 to $5000 \mathrm{~Hz}$ range.

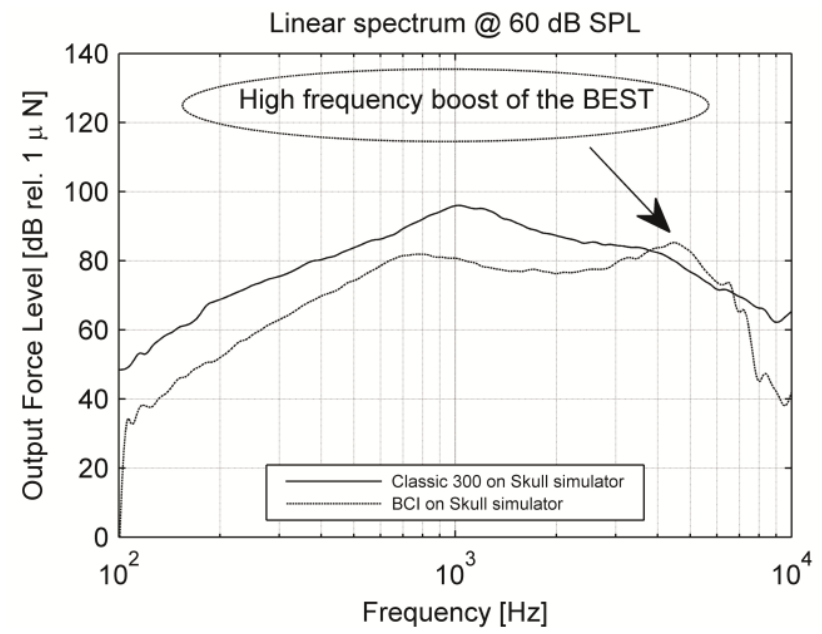

Figure 10. Linear spectrum magnitude of the $P B C D$ and the $B C l$ (4 mm skin) measured on Skull simulator in the anechoic chamber at $60 \mathrm{~dB}$ SPL.

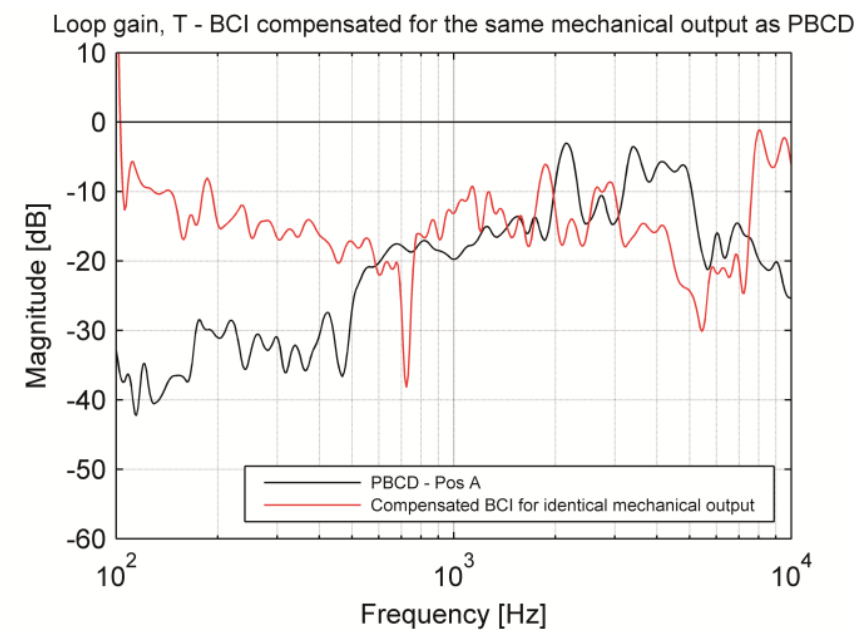


Figure 11. The $P B C D$ and the $B C l$ loop gain magnitudes on dry skull where the $B C l$ has been compensated to produce the same mechanical output as the PBCD.

\section{Compensated for the same cochlear acceleration level}

However, it has been shown in earlier studies $(15,16,21-23)$ on dry skull and cadaver heads that the sensitivity to bone-conducted sound would increase if the excitation point approaches the cochlea. Therefore, the $\mathrm{BCl}$ device should be compensated a second time by the gain differences between implantation in Pos A versus B. This was made by measuring the Promontory Acceleration Levels (PAL) using a laser Doppler vibrometer (LDV). The same PBCD transducer was attached in both positions on the dry skull. In Figure 12, the PAL for both positions are presented when 1 volt was applied to the transducer.

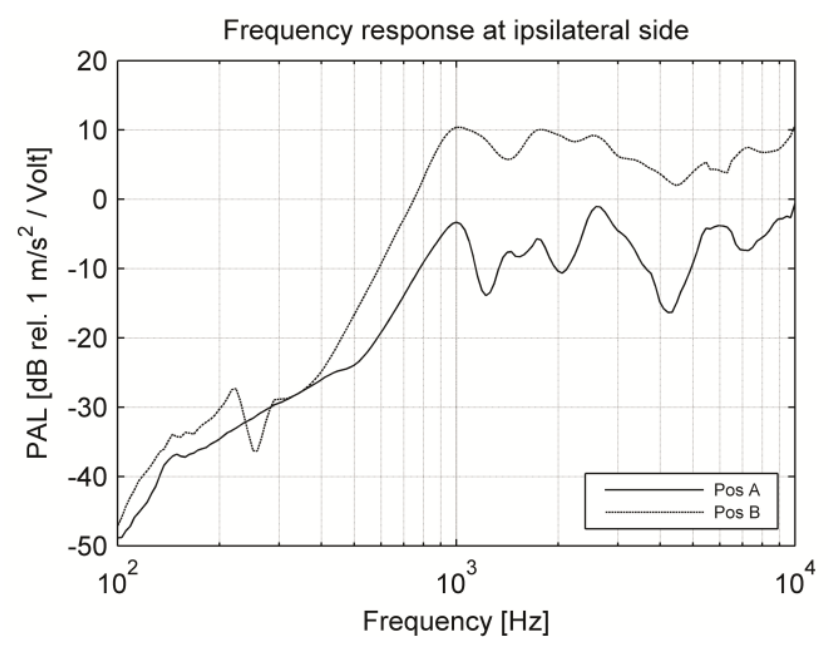

Figure 12. Frequency response magnitude (Promontory acceleration divided by input voltage) of the $P B C D$ transducer in Pos $A$ and Pos $B$ at the ipsilateral side on the dry skull.

Based on the PAL difference between the Position $A$ and Position $B$, the compensated $B C l$ loop gain in Figure 12 was once more compensated to yield the $\mathrm{BCl}$ loop gain presented in Figure 13. Now the $\mathrm{BCl}$ loop gain is also compensated to give the same cochlear acceleration as the PBCD which gives additional 0-25 dB more gain headroom as compared with the PBCD. It can be observed that the compensated $\mathrm{BCl}$ for the same cochlear acceleration level has 10-30 dB higher gain headroom than the PBCD in the range of 600 to $7500 \mathrm{~Hz}$. The gain headroom improvement at the critical frequency is $17 \mathrm{~dB}$. The improved gain headroom indicates how much the amplification can be increased with the $\mathrm{BCl}$ device, as compared with the PBCD, without obtaining feedback oscillations. Reviewing the call for improving the high frequency gain in the PBCD (which is now inherently limited by feedback) as 
compared with conventional air conduction devices this improved gain headroom with the $\mathrm{BCl}$ may show to be of great clinical value.

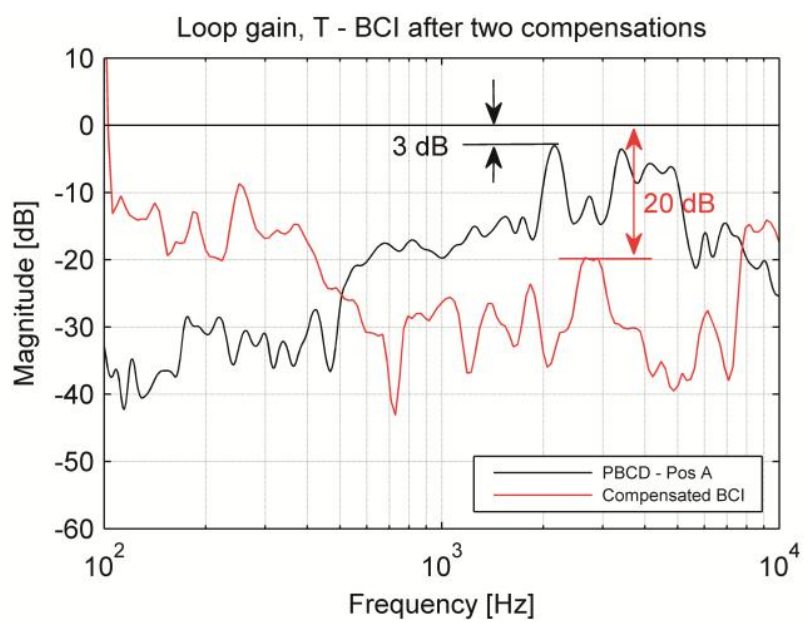

Figure 13. The $P B C D$ and the twice compensated $B C l$ loop gain magnitudes on dry skull. $B C l$ compensations were done to obtain the same cochlear acceleration level as the PBCD.

One relevant question to ask is why the difference in gain headroom between the $\mathrm{BCl}$ and the $\mathrm{PBCD}$ is so big. One possible explanation may be that the difference in mechanical point impedances in Pos A and B may play a role. It has been shown by Eeg-Olofsson et al. (23) that mechanical impedance is significantly higher in Position B as compared with Position A and hence the radiation of sound for a given force excitation might be lower in Position B than in Position A. Therefore, we repeated the loop gain measurements on the other side of the dry skull (right side) but this time with the same percutaneous bone conduction device in both position $A$ and $B$. To place the PBCD in Pos $B$, an extension rod was attached to the skull bone and the abutment was screwed on top of the rod. It was found that position $B$ has 1-5 dB more gain headroom in some frequencies than position $A$ and hence does not explain the big differences in loop gain between the PBCD and the $\mathrm{BCl}$ (Figure 13). Another explanation may be that the acoustic contribution of feedback is much higher in the PBCD than in the $\mathrm{BCl}$. This can be understandable as the transducer in the PBCD is in the same housing as the microphone whereas in the $\mathrm{BCl}$ they are separated and having skin and subcutaneous tissue in between. This explanation is further supported by the fact that the difference between the open and occluded microphone condition is 10-20 dB bigger for the PBCD (Figure 7) than for the $\mathrm{BCl}$ (Figure 8). Present findings are only based on measurement and analysis of one dry skull and it is of course very important to repeat these measurements on real patients when such are available. If other percutaneous bone conduction devices should be tested in terms of "loop gain" measurement, they should be linear, should not have any feedback cancelation enabled in the DSP and it should be 
possible to open/break the signal loop internally of the amplifier preferably after the preamplifier for noise reasons. This was possible in the Classic 300 , but it is not obviously possible in newer models with manufacture specific DSP. As the feedback path in the PBCD is dominated by acoustical feedback due to the skull radiation and transducer suspension/motion, the results from using Classic 300 may be generic and also valid for newer models.

\section{Conclusion}

A new bone conduction implant $(\mathrm{BCl})$ device which keeps the covering skin intact after healing has been developed as an alternative to the percutaneous bone conduction device. In cadavers studies it has been noticed that the $\mathrm{BCl}$ was less prone to fall into feedback problems. Hence the scope of this study was to investigate feedback in the $\mathrm{PBCD}$ and the $\mathrm{BCl}$. It was found that the $\mathrm{BCl}$ had an improved gain headroom of $10-30 \mathrm{~dB}$ versus the PBCD, if the mechanical output of the devices were normalized at the cochlear level. More specifically the improvement in gain headroom at the critical frequency was $17 \mathrm{~dB}$. One reason might be due to the higher mechanical point impedance of the position B compared with position A which imply that less sound is radiated for a given force level. Another explanation for this improved gain headroom might be that the transducer in the $\mathrm{BCl}$ is completely encapsulated and is mechanically separated from the microphone in the audio processor. The improved gain headroom will allow a possibility to increase the real gain of the $\mathrm{BCl}$ compared to the PBCD. This may have a significant clinical importance for the hearing rehabilitation of patients. In conclusion it was found that the $\mathrm{BCl}$ was less prone to fall into feedback problems than the PBCD but these tests should also be conducted on real patients to confirm the clinical benefits.

\section{References}

1. Snik AFM, Myllanus EM, Cremers C. The bone-anchored hearing aid compared with conventional hearing aids. Otolaryngol Clin North Am 1995;28: 73-78.

2. Tjellström A, Håkansson B, Granström $G$. The bone-anchored hearing aids-current status in adults and children. Otolaryngol Clin North Am 2001;34:337-64.

3. Snik AFM, Mylanus EAM, Proops DW, et al. Consensus statements on the BAHA system: where do we stand at present? Ann Otol Rhinol Laryngol Suppl 2005;195:2-12.

4. Stewart C.M., Clark J.H., Niparko J.K. Bone-Anchored Devices in Single-Sided. In: Kompis M, Caversaccio M-D. Implantable Bone Conduction Hearing Aids. Basel: Karger Press, 2011. $92-$ 102. 
5. Wazen JJ, Van Ess MJ, Alameda J, Ortega C, Modisett M, Pinsky K. The Baha System in Patients with Single-Sided Deafness and Contralateral Hearing Loss. Otolaryngol Head Neck Surg 2010;142:554-9.

6. Hol MK, Kunst SJ, Snik AF, Cremers CW. Pilot Study on the Effectiveness of the Conventional CROS, the Transcranial CROS and the BAHA Transcranial CROS in Adults with Unilateral Inner Ear Deafness. Eur Arch Otorhinolaryngol 2010; 267:889-96.

7. Tjellström A, Granström G. Long-term follow-up with the bone anchored hearing aid: a review of the first 100 patients between 1977 and 1985. ENT J 1994;73:21-3.

8. Reyes RA, Tjellström A, Granström G. Evaluation of implant losses and skin reaction around extraoral bone anchored hearing implants: a 0- to 8-year follow-up. Otolaryngol Head Neck Surg 2000;122:272-6.

9. Battista RA, Littlefield PD. Revision BAHA surgery. Otolaryngol Clin North Am 2006;39:801-13.

10. Shirazi M, Marzo S, Leonetti J. Perioperative complications with the bone anchored hearing aid. Otolaryngol Head Neck Surg 2006;134:236-9.

11. Wazen J, Wycherly B, Daugherty J. Complications of Bone-Anchored Hearing Devices. In: Kompis M, Caversaccio M-D. Implantable Bone Conduction Hearing Aids. Basel: Karger Press, 2011. 63-72.

12. Dun CA, de Wolf MJ, Hol MK, Wigren S, Eeg-Olofsson M, et al. Stability, Survival, and Tolerability of a Novel Baha Implant System:Six-Month Data from m Multicenter Clinical Investigation. Otol Neurotol 2011; 32:1001-7.

13. de Wolf MJ, Hol MK, Mylanus EA, Cremers CW. Bone-Anchored hearing Aid Surgery in Older Adults: Implant Loss and Skin Reactions. Ann Otol Rhinol Laryngol 2009;118:525-31.

14. de Wolf MJ, Hol MK, Huygen PL, Mylanus EA, Cremers CW. Nijmegen Results with Application of a Bone-Anchored Hearing Aid in Children: Simplified Surgical Technique. Ann Otol Rhinol Laryngol 2008;117:805-14.

15. Håkansson B, Eeg-Olofsson M, Reinfeldt S, et al. Percutaneous Versus Transcutaneous Bone Conduction Implant system: A Feasibility Study on a Cadaver Head. Otol Neurotol 2008;29:1132-1139.

16. Håkansson $B$, Reinfeldt $S$, Eeg Olofsson $M$, et al. A novel bone conduction implant (BCI): Engineering aspects and pre-clinical studies. Int J Audiol 2010;49:203-215.

17. Håkansson B. The Future of Bone Conduction Hearing Devices. In: Kompis M, Caversaccio MD. Implantable Bone Conduction Hearing Aids. Basel: Karger Press, 2011. 140-152.

18. Carlsson P. On Direct Bone Conduction Hearing Devices. Ph.D. thesis. Technical report number: 195/1990, Chalmers University of Technology, Göteborg, Sweden. 
19. Taghavi H, Håkansson B, Reinfeldt S, et al. A Novel Bone Conduction Implant (BCl) System. Presented at the $3^{\text {rd }}$ International Symposium on Bone Conduction Hearing - Craniofacial Osseointegration 2011, Sarasota, Florida, March 23-26, 2011.

20. Håkansson B, Carlsson P. Skull simulator for direct bone conduction hearing devices. Scandinavian Audiology 1989;18:91-8.

21. Stenfelt S, Håkansson B, Tjellström A. Vibration characteristics of bone conducted sound in vitro. J Acoust Soc Am 2000;107:422-31

22. Stenfelt S, Goode RL. Transmission properties of bone conducted sound: measurements in cadaver heads. J Acoust Soc Am 2005;118:2373-91.

23. Eeg-Olofsson M, Granström G, Stenfelt S, Tjellström A. Transmission of bone conducted sound in the human skull measured by cochlear vibrations. Int J Audiol 2008;47:1-9. 